\title{
Energy spectroscopy of controlled coupled quantum-wire states
}

\author{
S. F. FISCHER ${ }^{1 *}$, G. APETRII ${ }^{1}$, U. KUNZE ${ }^{1}$, D. SCHUH ${ }^{2 \dagger}$ AND G. ABSTREITER ${ }^{2}$ \\ ${ }^{1}$ Werkstoffe und Nanoelektronik, Ruhr-Universität Bochum, D-44780 Bochum, Germany \\ ${ }^{2}$ Walter Schottky Institut, Technische Universität München, D-85748 Garching, Germany \\ ${ }^{\dagger}$ Present address: Angewandte und Experimentelle Physik, Universität Regensburg, D-93040 Regensburg, Germany \\ *e-mail: saskia.fischer@rub.de
}

Q uantum ballistic transport in electron waveguides $(\mathrm{EWGs})^{1,2}$ is based on coherent quantum states arising from the one-dimensional (1D) confinement in nanometre-scale constrictions. Semiconductor EWGs have received considerable renewed interest for quantum logic devices ${ }^{3-7}$ and theoretical concepts $^{8-11}$ in the context of solid-state quantum information processing ${ }^{12}$. Implementation in real-world quantum circuits requires the unambiguous experimental distinction between all involved energy levels. However, such knowledge of EWGs investigated for wavefunction hybridization ${ }^{13-15}$ is solely based on estimates. Here, we present coupled EWGs that allow single-mode control and manipulation of mode coupling at temperatures as high as that of liquid-helium $(4.2 \mathrm{~K})$ and above. We demonstrate high-resolution energy spectroscopy of each EWG subband ladder and the $1 \mathrm{D}$ coupled states involved. The results verify the power of advanced nanolithography and its ability to open the door to the scalable semiconductor quantum circuits envisaged today.

Quantum 1D conductors formed from two-dimensional (2D) electron gases (2DEGs) are important in nanoscopic and mesoscopic semiconductor devices when studying the physics of coherent electron flow ${ }^{1,2}$. Spatial 1D constrictions are formed of the order of the Fermi wavelength $\lambda_{\mathrm{F}} \sim 35 \mathrm{~nm}$ and much below the mean free path length of $l \sim 10 \mu \mathrm{m}$ as achieved in high-electron-mobility $\left(\sim 1 \times 10^{6} \mathrm{~cm}^{2} \mathrm{~V}^{-1} \mathrm{~s}^{-1}\right)$ 2DEGs in $\mathrm{AlGaAs} / \mathrm{GaAs}$ heterostructures. Various techniques can be used, such as metal-deposited split gates ${ }^{16,17}$, etching ${ }^{18-20}$ or local anodic oxidation $^{21}$. Conductance quantization ${ }^{16,17}$ in linear and nonlinear transport is reported for etched single EWGs for temperatures up to $30 \mathrm{~K}$ (ref. 19), which requires large $1 \mathrm{D}$ subband separations $(>10 \mathrm{meV})$. So far, coupled EWGs have been restricted to 1D subband spacings of a few millielectronvolts, hampering the direct high-resolution energy spectroscopy of each single EWG and their coupled modes ${ }^{13-15}$. Only estimates of splitting energies are known ${ }^{13,22}$. Our objective here is to demonstrate direct high-resolution energy spectroscopy applicable to various coupled EWGs. The complete knowledge of mode-coupled 1D energy spectra is provided fulfilling a prerequisite for quantum engineering of proposed complex EWG devices ${ }^{3-11}$.
Nanolithography with an atomic force microscope (AFM, Digital Instruments, Nanoscope III) enables us to produce EWGs showing $1 \mathrm{D}$ subband spacings above $10 \mathrm{meV}$ allowing single-mode operation and control of mode coupling at liquid-helium temperature $(4.2 \mathrm{~K})$ and above.

Spatially separated coupled EWGs can be realized using a tunnel barrier, either from two vertically stacked 2DEGs or from a single 2DEG with a lateral tunnel barrier. Spatially coincident coupled EWGs can also be realized, by either injecting electrons from different modes of the $2 \mathrm{D}$ reservoirs or by laterally merging the electron flow from two EWGs into one junction. In each case, mode coupling occurs as wavefunction hybridization ${ }^{13}\left|\Psi_{+/-}\right\rangle=$ $a_{1}\left|\Psi_{s 1, n 1}\right\rangle \pm a_{2}\left|\Psi_{s 2, n 2}\right\rangle$, which results in splitting $\Delta E$ of two degenerate $1 \mathrm{D}$ energy levels $E_{n}^{s}$ (see Fig. 1d). Here, $s$ distinguishes between the EWGs and the quantum number $n$ denotes the $1 \mathrm{D}$ transverse mode index. The symmetry of the confining potential $V(x, y, z)$ defines the mode spectra ${ }^{23}$ including all experimentally observed level crossings and anticrossings ${ }^{13-15,22,24}$. In our study, the mixing between $n 1$ and $n 2$ subbands of the wires labelled $s 1$ and $s 2$ is determined by the matrix element $\left\langle\Psi_{s 1, n 1}|V(z)| \Psi_{s 2, n 2}\right\rangle$, where $V(z)$ is the conduction-band profile of the quantum well under consideration as detailed in ref. 13. Mode coupling is expected in aligned wires of equal width for transverse modes of $n 1=n 2$ and in aligned wires of different width if $n 1$ and $n 2$ are either both odd or even. In finite magnetic fields the interaction can be strongly modified (see below).

We fabricated spatially coincident and spatially separated coupled 1D electron systems. The devices are short EWGs in three-terminal AlGaAs/GaAs modulation-doped field-effect transistor structures (Fig. 1a). Ohmic source and drain contacts to the 2DEG reservoirs allow us to pass a source-drain current through the $1 \mathrm{D}$ channel. A Schottky-contact top gate covers the 2DEG reservoirs and the 1D channel. The barriers forming the 1D constriction are established by nanogrooves wet-etched into the semiconductor surface beneath which the $2 \mathrm{D}$ electron gas is locally depleted (Fig. 1b). Spatially coincident EWGs are formed from the ground and first excited states of a $2 \mathrm{DEG}$ in a 30 -nm-wide GaAs quantum well ${ }^{22}$ (see Supplementary Information, Fig. S1). Spatially separated tunnel-coupled EWGs, on the other hand, are 
a

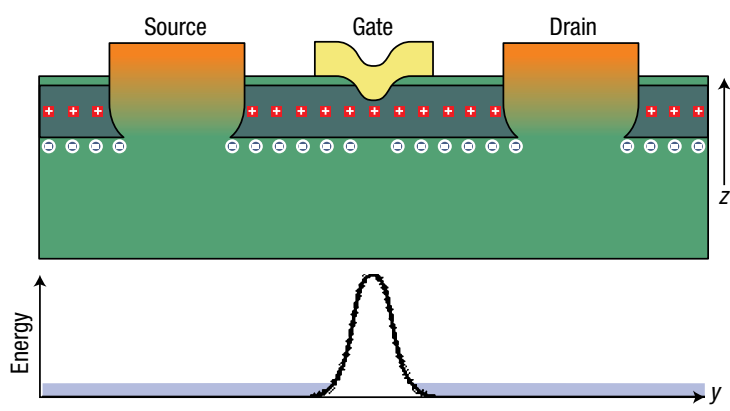

G

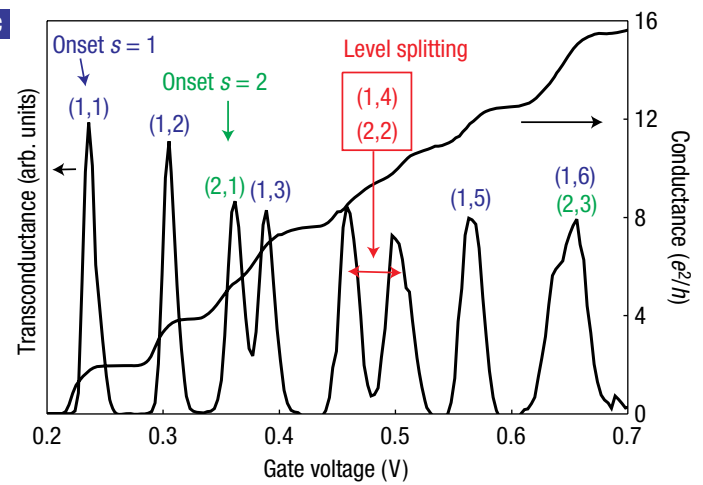

b
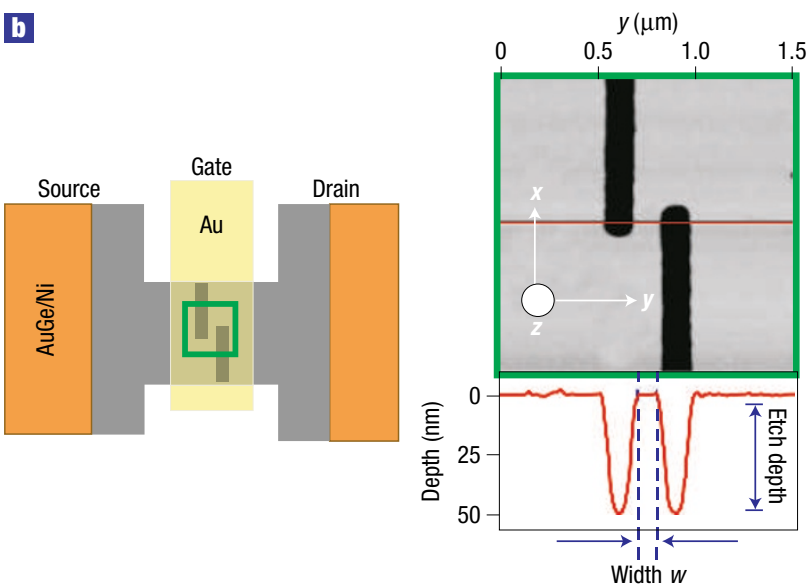

d

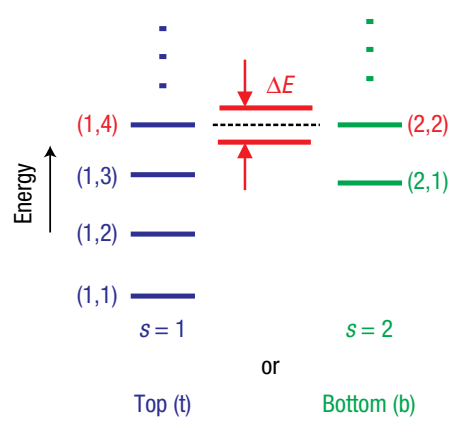

Figure 1 Schematic diagrams illustrating the main features of our EWG devices, the experimental system and exemplary two-terminal measurements and quantum-well band-edge calculations. a, Schematic illustration of the cross-sectional view of the device and potential landscape. Local barriers are formed by etched nanogrooves. $\mathbf{b}$, Schematic illustration of the device top view and AFM image of the nanogrooves forming the 1D constriction taken before top-gate deposition. c, Conductance and transconductance measurements with mode indices for a 125-nm-wide EWG. d, Schematic illustration of energy splitting of degenerate 1D subband edges in the case of mode coupling.

based on two vertically stacked $2 \mathrm{D}$ electron systems hosted in a 30-nm-wide GaAs quantum well with $1 \mathrm{~nm}$ of AlGaAs barrier in its middle (see Supplementary Information, Fig. S1). Details of the heterostructures and the measurements are given in the Methods section.

Coupled EWGs show deviations from the normal conductance increase $e^{22,24,25}$ quantized by $2 e^{2} / h$ ( $e$ is the electron charge and $h$ Planck's constant, see Fig. $1 c$ and Supplementary Information, Fig. S1), revealing that two $1 \mathrm{D}$ subband edges are in close proximity. Double conductance steps of $4 e^{2} / h$ are formed if $1 \mathrm{D}$ levels become degenerate. The contributing modes can unambiguously be identified by shifting the subladders relative to each other either by purely electrostatic means ${ }^{24}$ or by magnetotransport spectroscopy ${ }^{22}$. Mode coupling has been observed in terms of level anticrossings in the transconductance ${ }^{13-15,22}$.

Energy spectroscopy of mode coupling is demonstrated for spatially coincident EWGs first. The two 1D electron systems arising from the occupation of the ground and first excited states of 2D reservoirs (see Supplementary Information, Fig. S1) are depicted as the first $(s=1)$ and second $(s=2)$ vertical mode, respectively (Fig. 1d). The $1 \mathrm{D}$ electron wavefunctions can be denoted as $\Psi_{s, n}(x, y, z)=\exp \left(i k_{x} x\right) \psi_{s, n}(y, z)$ with directions $x$ for propagating modes, $y$ and $z$ for lateral and vertical confinement, respectively. For decoupled lateral and vertical components, the envelope wavefunction $\psi_{s, n}(y, z)=\varphi_{n}(y) \Phi_{s}(z)$, where $\varphi_{n}(y)$ denotes the $n$th lateral mode and $\Phi_{s}(z)$ the vertical wavefunction of the quantum well. In-plane magnetic fields $B$ energetically shift the two $1 \mathrm{D}$ subbladders, $E_{n}^{1}$ and $E_{n}^{2}$, relative to each other ${ }^{22}$. In transverse magnetic fields, see Fig. 2a, 1D subbands for $s=2$ experience a stronger diamagnetic shift ${ }^{26}$ proportial to $B^{2}$ than for $s=1$ on behalf of larger spatial extensions of the vertical wavefunction $^{22}$ (see Supplementary Information, Fig. S2). Mode coupling between wires of slightly different lateral confinement occurs for $1 \mathrm{D}$ levels of equal parity in the transverse mode index ${ }^{13,22}$ $n$ (see Supplementary Information, Fig. S4). In longitudinal magnetic fields, see Fig. 2b, lateral and vertical components of the confining potential are coupled leading to Fock-Darwin-like spectra $^{14,22}$ and complex mode mixing ${ }^{13}$.

A relative shift of $1 \mathrm{D}$ subladders can be performed purely electrostatically by cooling the sample under gate bias ${ }^{22}$. Here, the confining potential is varied by persistent recharging of the doping layer $^{27}$ (see Supplementary Information, Fig. S3). This enables the observation of coincidences of different pairs of modes in the magnetotransport spectra ${ }^{22,24}$ because of a shifted subladder onset.

Recording the quantized conductance under d.c. source-drain bias allows direct imaging of mode coupling and spectroscopy of the corresponding energy splittings as demonstrated in Fig. $2 \mathrm{c}$ and $\mathrm{d}$. The transconductance maxima (in black) reflect coincidences of the chemical potential $\mu_{\mathrm{S}, \mathrm{D}}$ of the source or drain reservoir with $1 \mathrm{D}$ subband edges $E_{n}^{s}$. For a single EWG, 

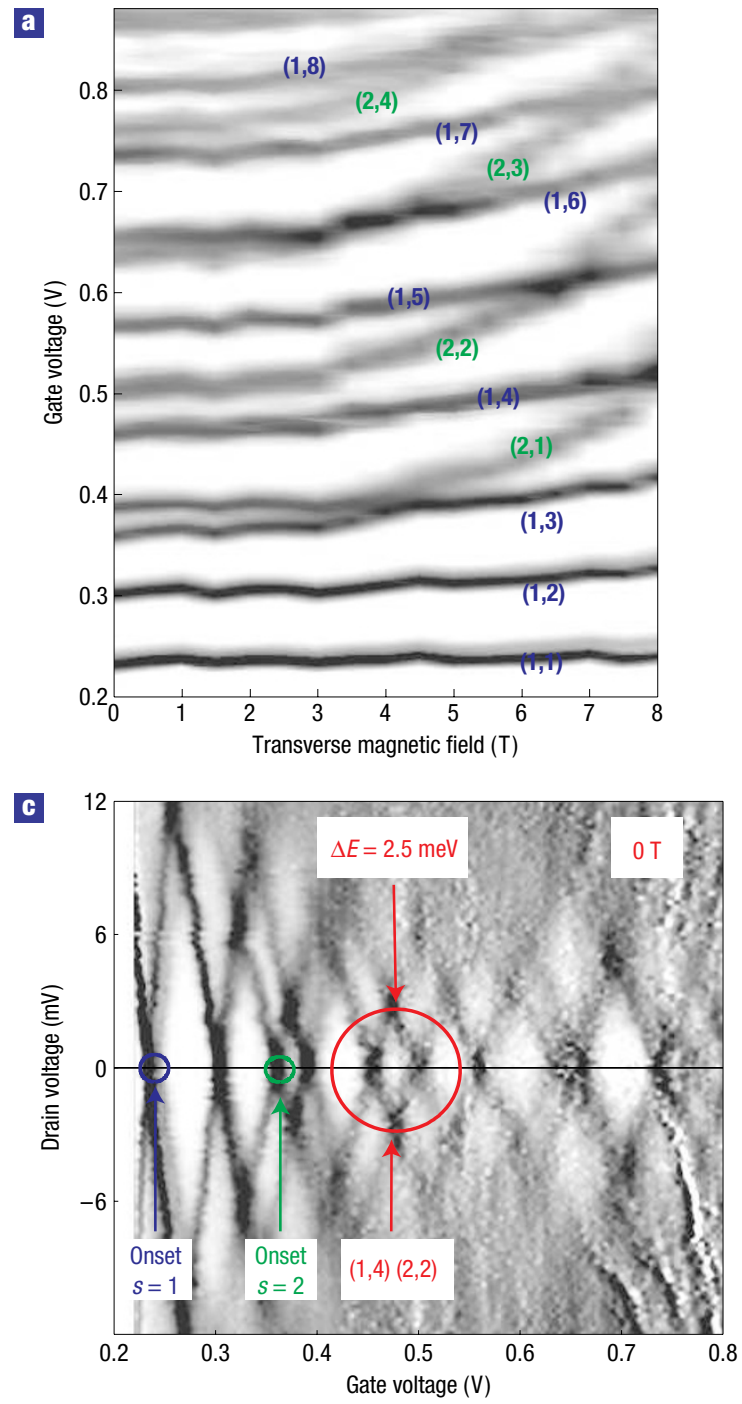
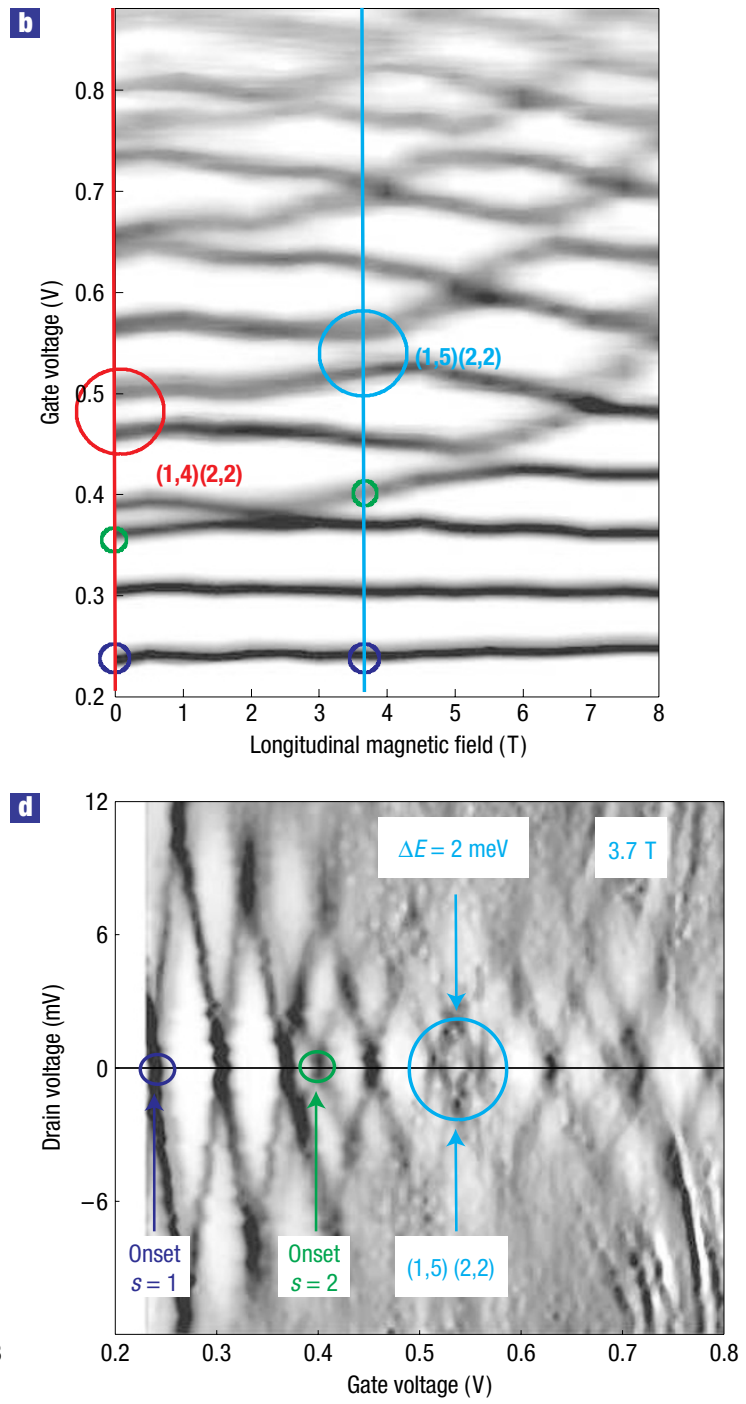

Figure 2 In-plane magnetotransport and bias spectra of the transconductance for a 125-nm-wide constriction hosting two spatially coincident 1D electron systems at $2 \mathrm{~K}$. a,b, No cooling bias $\left(V_{\mathrm{c}}=0 \mathrm{~V}\right)$ : transverse applied magnetic field (a) and longitudinal applied magnetic field (b). c,d, Bias spectroscopy at fixed longitudinal magnetic fields from $\mathbf{b}$, at $0 \mathrm{~T}(\mathbf{c})$ and $3.7 \mathrm{~T}(\mathbf{d})$.

extensions of the rhombic patterns of transconductance maxima on the drain bias voltage scale are a measure of energy separations $\Delta E_{n, n+1}^{s}$ between subsequent $1 \mathrm{D}$-subband edges ${ }^{28}$. For coupled EWGs, two superimposed sequences of rhombic pattern depict the two subladders. Furthermore, e patterns can appear as a consequence of mode coupling. Comparing Fig. 2d with the corresponding magnetotransport spectrum in Fig. $2 \mathrm{~b}$ also leads to an unambiguous identification of the onset of the second 1D subladder for each applied magnetic field. Level anticrossings evident in the mode spectrum, such as $(1,5)$ and $(2,2)$ at $B=3.7 \mathrm{~T}$ encircled in Fig. $2 \mathrm{~b}$, are directly reflected in the bias spectroscopy (Fig. 2d, encircled). Here, the energy splitting $\Delta E=2 \mathrm{meV}$ of the coupled modes $(1,5)$ and $(2,2)$ depicts an intermediate coupling strength when compared with the 1D subband spacing $\Delta E_{1,2}^{1}=10 \mathrm{meV}$ of the first vertical mode. At zero magnetic field, energy spectroscopy helps to identify anticrossings: unphysical increases in subsequent subband spacings are observed in the independent single-wire subladder if uncoupled modes are assumed (an example is given in Supplementary Information,
Fig. S4). Furthermore, bias spectroscopy enables us to untangle different mode contributions as visible by the relative shift of the $1 \mathrm{D}$ subladders obtained by cooling under different gate bias voltages in Fig. 3a,b. Here, anticrossings such as $(1,4)$ and $(2,2)$ (Fig. 3a) disappear and a superimposed image of the two independent, undisturbed 1D subladders becomes visible (Fig. 3b). Detailed evaluation of bias spectroscopy allows the verification of assumptions made about each EWG confining potential (an example is given in Supplementary Information, Fig. S5).

One-dimensional subband spacings of more than $10 \mathrm{meV}$ allow us to operate EWGs in direct contact with a liquid-helium bath, which is favourable for any long-term measurement. Figure 3a,b demonstrates high-resolution energy spectroscopy at $4.2 \mathrm{~K}$. Figure $3 \mathrm{a}$ corresponds to the $2 \mathrm{~K}$ data in Fig. 2c. The anticrossing can be resolved up to $5.2 \mathrm{~K}$, see Fig. $3 \mathrm{~d}$.

Tunnel-coupled EWGs are of much interest for application in quantum device circuits ${ }^{3,4,6-11}$. In our vertically stacked EWGs of symmetric quantum wells, the 1D sublevels of the bottom EWG $(\mathrm{b}, n)$ vary more strongly with applied back gate voltage 

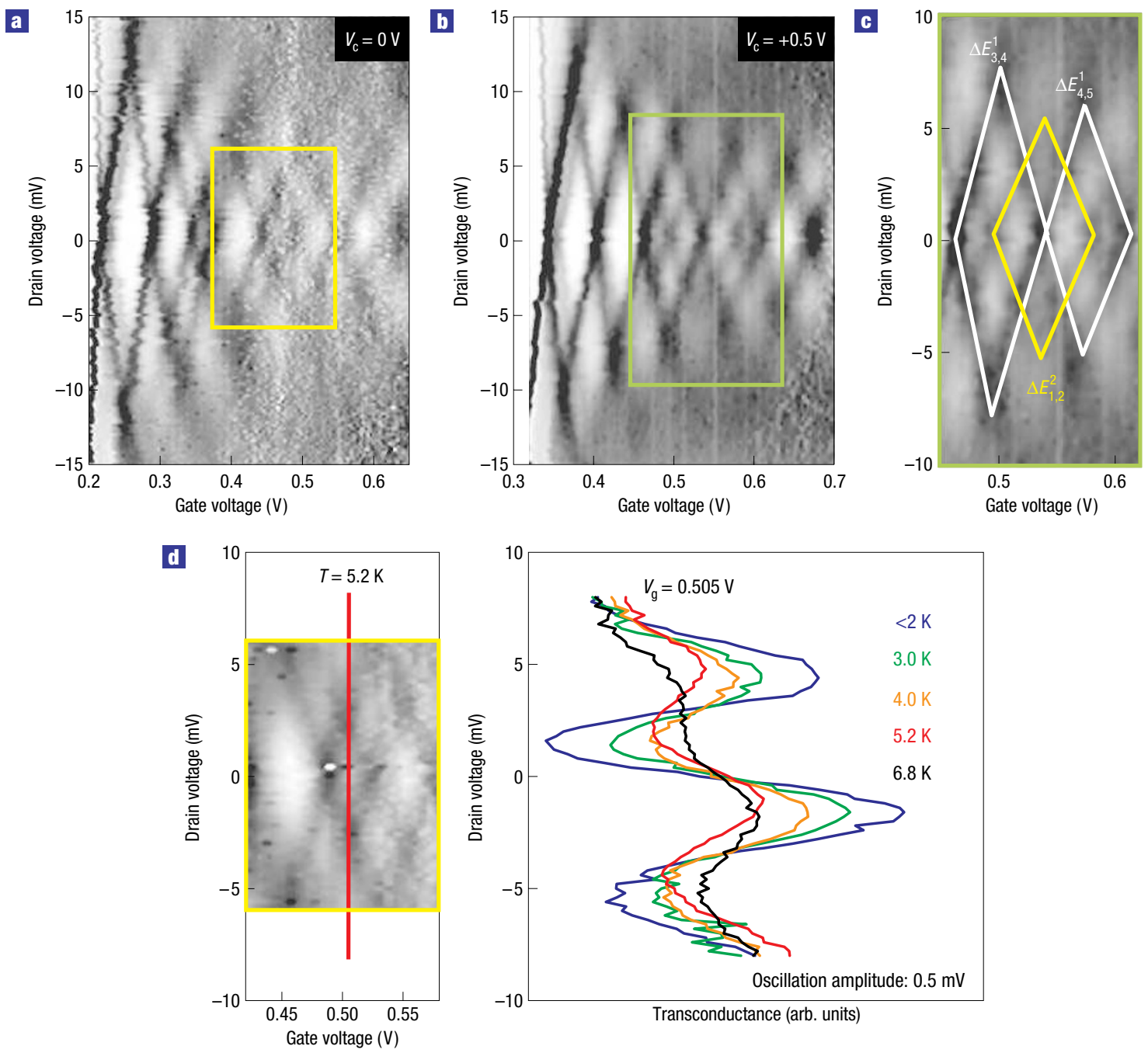

Figure 3 Bias spectroscopy of a 125-nm-wide constriction hosting two spatially coincident $1 \mathrm{D}$ electron systems at elevated temperatures. a,b, $T=4.2 \mathrm{~K}$ for different cooling bias voltages $V_{c}: 0 \mathrm{~V}(\mathbf{a})+0.5 \mathrm{~V}(\mathbf{b})$. c, Enlargement of the coexistence region of undisturbed superimposed energy spectra of both vertical modes from $\mathbf{b}$. d, Enlargement of the anticrossing region in a taken at $T=5.2 \mathrm{~K}$ (left) and the temperature dependence of measured transconductance lines at the anticrossing.

$V_{\mathrm{bg}}$ than levels from the top EWG $(\mathrm{t}, n)$ owing to electrostatic screening. Therefore, a purely electrostatic shift of $1 \mathrm{D}$ subladders is possible. Degenerate $1 \mathrm{D}$-subband edges of unequal transverse mode index $n$ lead to level crossing with varied backgate voltage $e^{13}$ (see Supplementary Information, Fig. S6). Mode coupling occurs for 1D levels from the bottom $(s=\mathrm{b})$ and top $(s=\mathrm{t})$ EWG with equal transverse mode indices ${ }^{13} n$. This special situation is fulfilled for EWGs of nearly equal threshold as depicted by the greyscale plot of $4.2 \mathrm{~K}$ transconductance measurements versus top and back gate voltage in Fig. 4a for different cooling biases. Bias spectroscopy at $V_{\text {bg }}=-210 \mathrm{~V}$ (Fig. 4c) and $210 \mathrm{~V}$ (Fig. 4e) enables us to determine energy separations between the first and second 1D subband of the top and bottom EWGs to 12.3 and $10.8 \mathrm{meV}$, respectively. Furthermore, at zero back gate voltage (Fig. 4d) for which mode coupling occurs, bias spectroscopy reveals splitting energies of 5.4, 3.9 and $2.9 \mathrm{meV}$ for $n=1,2$ and 3, respectively. Mode coupling is seen for temperatures above $10 \mathrm{~K}$. In Fig. $4 \mathrm{~b}$, the transconductance peaks of the split levels are unambiguously distinguishable.

On increasing temperature $T$ the conductance plateaux in single EWGs acquire a finite slope $e^{1,2}, G\left(E_{\mathrm{F}}, T\right)=$ $\int_{0}^{\infty} G(E, 0)\left(\mathrm{d} f / \mathrm{d} E_{\mathrm{F}}\right) \mathrm{d} E=2 e^{2} / h \sum_{n=1}^{\infty} f\left(E_{n}-E_{\mathrm{F}}\right)$, as the FermiDirac distribution $f\left(E-E_{\mathrm{F}}\right)=\left(1+\exp \left(E-E_{\mathrm{F}} / k_{\mathrm{B}} T\right)\right)^{-1}$ becomes smeared ( $E_{\mathrm{F}}$ is the Fermi energy and $k_{\mathrm{B}}$ is the Boltzmann constant). The observed energy resolution $\Delta E$ corresponds to the width of the thermal broadening $\mathrm{d} f / \mathrm{d} E_{\mathrm{F}}$ of $\sim 4 k_{\mathrm{B}} \mathrm{T}$. As our measurements of coupled EWGs show, subband spacings of $10 \mathrm{meV}$ are also resolved up to $20 \mathrm{~K}$ and level splittings of $2.5 \mathrm{meV}$ are accessible up to $5 \mathrm{~K}$. The thermal dephasing time $\tau_{\mathrm{th}}=\pi \hbar / k_{\mathrm{B}} T$ (where $\hbar$ is the reduced Planck constant) decreases from $6 \mathrm{ps}$ at $4.2 \mathrm{~K}$ to $2.4 \mathrm{ps}$ at $10 \mathrm{~K}$ and is the dominating timescale in our heterostructures above $4 \mathrm{~K}$. In comparison, the scattering time $\tau_{\mathrm{sc}}=m^{*} \mu / e$ is $32 \mathrm{ps}$ for the two subband 2DEGs and 9 ps for the vertically stacked 2DEGs, where $m^{*}$ is the effective mass and $\mu$ the electron mobility. Phasecoherence times $\tau_{\mathrm{ph}}$ of about 60 ps ensure the so-called coherent quantum-wire states in single EWGs ${ }^{1,2,29}$. Superposition states formed by coupling require coherence in the transverse modes. They lead to splitting of otherwise degenerate 1D subband edges and are experimentally detectable by means of bias spectroscopy.

As we have demonstrated, level spectroscopy and identification of single and coupled quantum-wire states can be established 

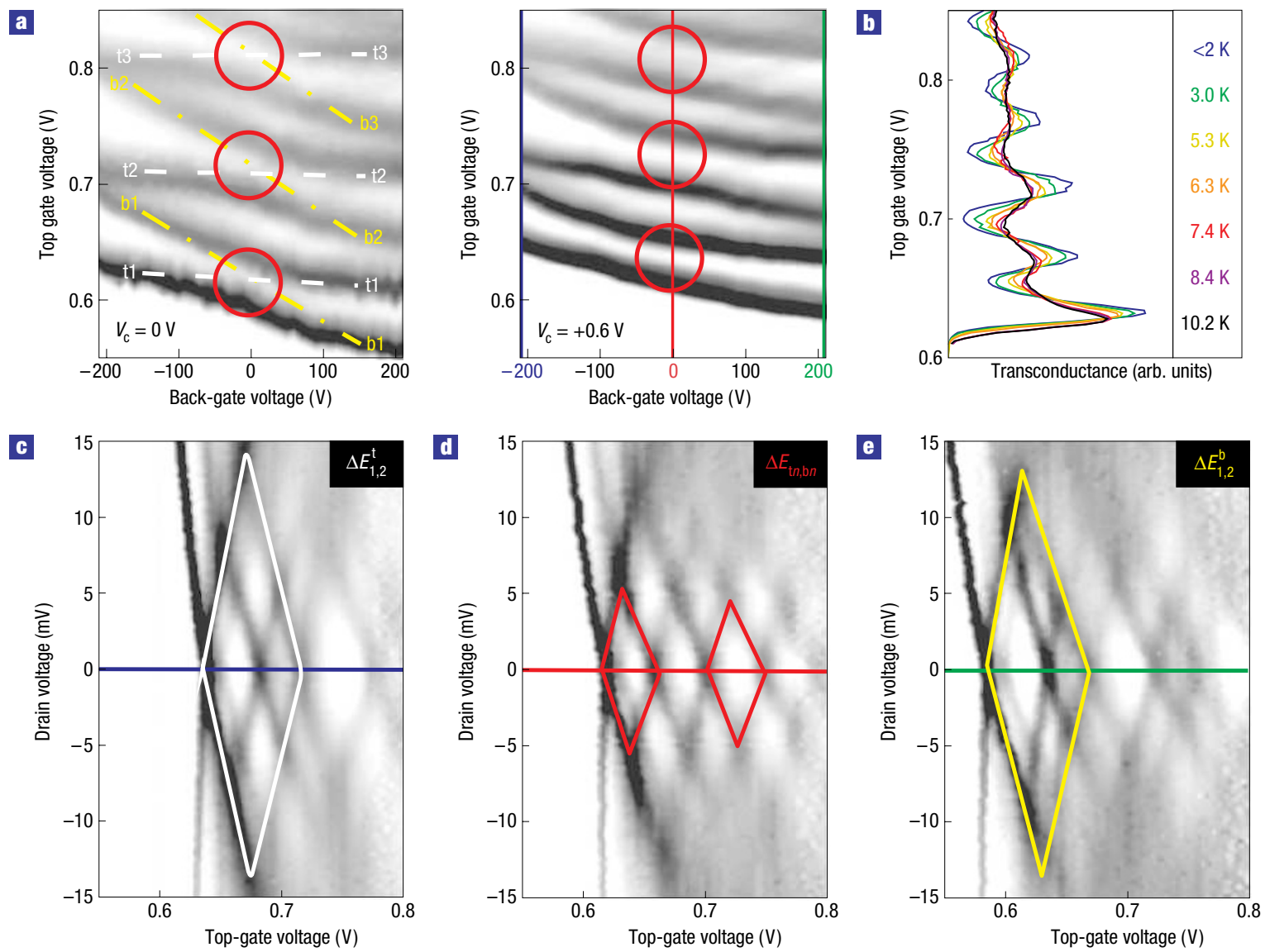

Figure 4 Transconductance bias spectra of a 130-nm-wide constriction hosting two tunnel-coupled vertically stacked 1D electron systems at $T=4.2 \mathrm{~K}$. a, Mode spectra of measured transconductance versus top gate and back gate voltages $V_{\mathrm{bg}}$ with cooling bias $V_{\mathrm{c}}=0 \mathrm{~V}$ (left) and $+0.6 \mathrm{~V}$ (right). Anticrossings of top (t) and bottom (b) transverse mode occur at $V_{\mathrm{bg}}=0 \mathrm{~V}$. b. Temperature dependence of measured transconductance at $V_{\mathrm{bg}}=0 \mathrm{~V}\left(V_{\mathrm{c}}=+0.6 \mathrm{~V}\right)$. c-e, Bias spectroscopy at different back gate voltages: $V_{\mathrm{bg}}=-210 \mathrm{~V}(\mathbf{c}) ; V_{\mathrm{bg}}=0 \mathrm{~V}(\mathbf{d}) ; V_{\mathrm{bg}}=+210 \mathrm{~V}(\mathbf{e})$. The energy spacings determined are subject to a correction by the series resistances of 2,330, 1,100 and $800 \Omega$, respectively.

by all-electrical means. This paves the way for quantum engineering of coupled EWG devices. Further, we have shown that the manipulation and the control of coupled 1D modes is feasible at temperatures of liquid helium and above. This opens the field of research to standard low-temperature laboratory equipment. Widely accessible nanopatterning techniques may be used to prepare coupled EWGs: local probe techniques, electronbeam lithography and large-scale imprint lithography. Possible applications of multiple EWGs are bi-directional couplers ${ }^{3,4}$, quantum waveguide inverters ${ }^{5}$ and, for an all-electrical control of coherent quantum-wire superposition states, quantum networks capable of processing quantum information ${ }^{8-12}$.

\section{METHODS}

Two series of EWGs were fabricated from GaAs/AlGaAs heterostructures with different quantum wells. Spatially coincident EWGs were formed from a 30-nm-wide GaAs square quantum well situated $60 \mathrm{~nm}$ below the sample and Si delta-doped from both sides. The as-grown carrier mobility was determined in the dark as $8.5 \times 10^{5} \mathrm{~cm}^{2} \mathrm{~V}^{-1} \mathrm{~s}^{-1}$ at a density of $4.2 \times 10^{11} \mathrm{~cm}^{-2}$ at $4.2 \mathrm{~K}$. Spatially separated, tunnel-coupled EWGs were made from two 14.5 -nm-wide GaAs layers separated by a 1-nm-thick $\mathrm{Al}_{0.32} \mathrm{Ga}_{0.68}$ As barrier. The upper interface of the top quantum well lies $60 \mathrm{~nm}$ below the heterostructure surface. A Si delta-doped supply layer is situated on each side of the double quantum well. A sheet electron density of $4.3 \times 10^{11} \mathrm{~cm}^{-2}$ and a mobility of $2.4 \times 10^{5} \mathrm{~cm}^{2} \mathrm{~V}^{-1} \mathrm{~s}^{-1}$ were measured in the dark at $4.2 \mathrm{~K}$ for the structure without a top gate.

One-dimensional constrictions were defined introducing local lateral barriers by means of etched nanogrooves. Dynamic ploughing of a 7-nm-thick resist with an AFM and subsequent wet-chemical etching of a line pattern as shown in Fig. 1c lead to the complete depletion of electrons underneath in each heterostructure ${ }^{20}$. Two-terminal differential conductance and transconductance measurements were performed by means of a standard lock-in technique. The source-drain excitation voltage was $0.3 \mathrm{mV}$ r.m.s. at $433 \mathrm{~Hz}$. Series resistances were determined from the deviation of the conductance from the fundamental values. In addition, for transconductance measurements, the top-gate voltage was modulated with a $3 \mathrm{mV}$ r.m.s. voltage. The error for energy-level separations determined by source-drain d.c. bias spectroscopy amounts to $0.5 \mathrm{meV}$.

The conduction band edges and charge distribution of the quantum wells were obtained with the numerical simulation program AQUILA (ref. 30). The quantum-well width and barrier thickness were taken from the growth parameters as described above; the Fermi energy was assumed to be pinned by surface states at the middle of the bandgap. The heterostructure doping was chosen such that the $2 \mathrm{D}$ subband electron densities match the measurement results (see above) and are determined with donor concentrations of $N_{\mathrm{d}, \text { top }}=2.1 \times 10^{12} \mathrm{~cm}^{-2}$ and $N_{\mathrm{d} \text {, bottom }}=2.9 \times 10^{11} \mathrm{~cm}^{-2}$ for the top and bottom delta-doping layers, respectively, and $N_{\mathrm{A}}=10^{15} \mathrm{~cm}^{-3}$ for the acceptor concentration of GaAs. The latter results from p-type carbon doping unintentionally introduced during growth. 
Received 16 September 2005; accepted 30 November 2005; published 15 January 2006.

\section{References}

1. Beenakker, C. W. J. \& van Houten, H. Quantum transport in semiconductor nanostructures. Solid State Phys. 44, 1-228 (1991).

2. Ferry, D. K. \& Goodnick, S. M. Transport in Nanostructures (Cambridge Univ. Press, Cambridge, 1997).

3. Eugster, C. C., del Alamo, J. A., Rooks, M. J. \& Melloch, M. R. One-dimensional to one-dimensional tunnelling between electron wave-guides. Appl. Phys. Lett. 64, 3157-3159 (1994).

4. Tsukada, N., Wieck, A. D. \& Ploog, K. Proposal of novel electron wave coupled devices. Appl. Phys. Lett. 56, 2527-2529 (1990).

5. Gilbert, M. J., Akis, R. \& Ferry, D. K. Magnetically and electrically tunable semiconductor quantum waveguide inverter. Appl. Phys. Lett. 81, 4284-4286 (2002).

6. Pingue, P. et al. Coulomb blockade directional coupler. Appl. Phys. Lett. 86, 052102 (2005).

7. Bielejec, E., Seamons, J. A., Reno, J. L. \& Lilly, M. P. Tunneling and nonlinear transport in a vertically coupled GaAs/AlGaAs double quantum wire system. Appl. Phys. Lett. 86, 083101 (2005).

8. Kitagawa, M. \& Ueda, M. Nonlinear-interferometric generation of number-phase-correlated fermion states. Phys. Rev. Lett. 67, 1852-1854 (1991).

9. Bertoni, A., Bordone, P., Brunetti, R., Jacoboni, C. \& Reggiani, S. Quantum logic gates based on coherent electron transport in quantum wires. Phys. Rev. Lett. 84, 5912-5915 (2000).

10. Ioniciou, R., Amaratunga, G. \& Udrea, F. Quantum computation with ballistic electrons. Int. J. Mod. Phys. B 15, 125-133 (2001).

11. Snyder, M. G. \& Reichl, L. E. Ballistic electron wave guide adder. Phys. Rev. A 70, 052330 (2004).

12. Bennett, C. H. \& DiVincenzo, D. P. Quantum information and computation. Nature 404, $247-255$ (2000).

13. Thomas, K. J. et al. Controlled wave-function mixing in strongly coupled one-dimensional wires. Phys. Rev. B 59, 12252-12255 (1999).

14. Salis, G. et al. Mode spectroscopy and level coupling in ballistic electron waveguides. Phys. Rev. B 60 7756-7759 (1999).

15. Friedland, K. J., Saku, T., Hirayama, Y. \& Ploog, K. H. Electron coupling in weakly and strongly coupled quantum point contacts. Physica E 11, 144-148 (2001).

16. van Wees, B. J. et al. Quantized conductance of point contacts in a two-dimensional electron gas. Phys. Rev. Lett. 60, 848-850 (1988).

17. Wharam, D. A. et al. One dimensional transport and the quantisation of the ballistic resistances. J. Phys. C 21, L209-L214 (1988).

18. Takagaki, Y., Gamo, K., Namba, S., Takaoka, S. \& Murase, K. Quantized resistance in in-plane gated narrow constriction fabricated by wet etching. Appl. Phys. Lett. 57, 2916-2918 (1990)
19. Kristensen, A. et al. Conductance quantization above $30 \mathrm{~K}$ in GaAlAs shallow-etched quantum point contacts smoothly joined to the background 2DEG. J. Appl. Phys. 83, 607-609 (1998).

20. Apetrii, G., Fischer, S. F., Kunze, U., Reuter, D. \& Wieck, A. D. Influence of processing parameters on the transport properties of quantum point contacts fabricated with an atomic force microscope. Semicond. Sci. Technol. 17, 735-738 (2002).

21. Curson, N. J. et al. Ballistic transport in a GaAs $/ \mathrm{Al}_{\mathrm{x}} \mathrm{Ga}_{1-\mathrm{x}} \mathrm{As}$ one-dimensional channel fabricated using an atomic force microscope. Appl. Phys. Lett. 78, 3466-3468 (2001).

22. Fischer, S. F., Apetrii, G., Kunze, U., Schuh, D. \& Abstreiter, G. Magnetotransport spectroscopy of spatially coincident coupled electron waveguides. Phys. Rev. B 71, 195330 (2005).

23. Merzbacher, E. Quantum Mechanics 2nd edn 428-429 (Wiley, New York, 1970).

24. Apetrii, G., Fischer, S. F., Kunze, U., Schuh, D. \& Abstreiter, G. Vertical-mode dependence of coupling between an electron waveguide and reservoirs with two occupied subbands. Physica E 22, 398-401 (2004)

25. Büttiker, M. Quantized transmission of a saddle-point constriction. Phys. Rev. B 41, 7906-7909 (1990)

26. Stern, F. \& Howard, W. E. Properties of semiconductor surface inversion layers in the electric quantum limit. Phys. Rev. B 163, 816-835 (1967).

27. Fischer, S. F. et al. Control of the confining potential in ballistic constriction using a persistent charging effect. Appl. Phys. Lett. 81, 2779-2781 (2002).

28. Patel, N. K. et al. Evolution of half plateaus as a function of electric field in a ballistic quasi-one-dimensional constriction. Phys. Rev. B 44, 13549-13555 (1991).

29. Topinka, M. A. et al. Imaging coherent electron flow from a quantum point contact. Science $\mathbf{2 8 9}$, 2323-2326 (2000).

30. Rother, M. <http://www.mathworks.com/matlabcentral>.

\section{Acknowledgements}

Part of this work was supported by the Bundesministerium für Bildung und Forschung under grant no. 01BM920. G.A. gratefully acknowledges the financial support of the foundation Isolde Dietrich. S.F.F. is grateful to Y. Milev, A. Rüdinger, L. Murokh, C. van der Wal and R. Akis for valuable discussions.

Correspondence and requests for materials should be addressed to S.F.F.

Supplementary Information accompanies this paper on www.nature.com/naturephysics.

Competing financial interests

The authors declare that they have no competing financial interests.

Reprints and permission information is available online at http://npg.nature.com/reprintsandpermissions/ 